\title{
A educação em saúde indígena na formação de profissionais ambientados com o meio cultural: uma revisão bibliográfica
}

Indigenous health education in the training of professionals in the cultural environment: a literature review

Educación en salud indígena en la formación de profesionales en el entorno cultural: una revisión bibliográfica

Lázaro Saluci Ramos ${ }^{1 *}$, Ana Quésia Souza de Almeida², Wyara Ribeiro Pereira Oréchio², Mábila de Souza Gomes², Camila Machado de Oliveira²$^{2}$, Cláudia Márcia Corrêa de Jesus², Josete Silva Henrique Batista², Ivanete Alves Baptista², Lídia Barreto Cordeiro².

\section{RESUMO}

Objetivo: Reunir a bibliografia acerca das políticas de educação em saúde de forma que possam exercer a função da melhor maneira possível sem interferir ou ter seu trabalho prejudicado pelas diferenças culturais presentes nas tribos. Revisão bibliográfica: A revisão em questão tem seu campo de exploração presente na literatura e apresenta uma reunião de ideias que apontam a necessidade de uma educação reforçada em saúde indígena, à fim de garantir a preservação e a não violação da cultura local. Trata-se de garantia constitucional à saúde, um dos direitos básicos diretamente ligado ao principal bem jurídico protegido pela lei brasileira, a vida. Para tanto, os aspectos básicos da educação em saúde e seus objetivos nobres pautados nos direitos humanos e na proteção da memória e conservação da cultura, são objetos de estudo da presente revisão. Considerações finais: $O$ estudo expôs uma crítica acerca da realidade brasileira dos últimos séculos que, completamente invasiva e eurocêntrica, cristaliza a necessidade de propor uma reforma à educação em saúde à fim de contemplar o exercício da saúde pública nas aldeias indígenas sem ferir os preceitos culturais locais.

Palavras-chave: Educação em saúde, Saúde indígena, Direito fundamental à saúde.

\begin{abstract}
Objective: Gather the bibliography on health education policies so that they can exercise their function in the best possible way without interfering or having their work hampered by the cultural differences present in the tribes. Bibliographic review: The review in question has its field of exploration present in the literature and presents a collection of ideas that point to the need for reinforced education in indigenous health, in order to guarantee the preservation and non-violation of local culture. It is a constitutional guarantee to health, one of the basic rights directly linked to the main legal asset protected by Brazilian law, life. For this purpose, the basic aspects of health education and its noble objectives based on human rights and the protection of memory and conservation of culture, are objects of study in this review. Final considerations: The study exposed a criticism about the Brazilian reality of the last centuries that, completely invasive and Eurocentric, crystallizes the need to propose a reform to health education in order to contemplate the exercise of public health in indigenous villages without hurting local cultural precepts.
\end{abstract}

Key words: Health education, Indigenous health, Fundamental right to health.

${ }^{1}$ Faculdade Multivix (MULTIVIX), Cachoeiro de Itapemirim - ES. * E-mail: lazaro321123@gmail.com

${ }^{2}$ Faculdade Vale do Cricaré (FVC), São Mateus - ES.

SUBMETIDO EM: $8 / 2020$

ACEITO EM: 9/2020

PUBLICADO EM: 12/2020 
RESUMEN

Objetivo: Recopilar la bibliografía sobre políticas de educación para la salud para que puedan ejercer su función de la mejor manera posible sin interferir o ver obstaculizado su trabajo por las diferencias culturales presentes en las tribus. Revisión bibliográfica: la revisión en cuestión tiene su campo de exploración presente en la literatura y presenta una colección de ideas que apuntan a la necesidad de una educación reforzada en la salud indígena, a fin de garantizar la preservación y la no violación de la cultura local. Es una garantía constitucional para la salud, uno de los derechos básicos directamente vinculados al principal activo legal protegido por la ley brasileña, la vida. Para este propósito, los aspectos básicos de la educación para la salud y sus nobles objetivos basados en los derechos humanos y la protección de la memoria y la conservación de la cultura, son objetos de estudio en esta revisión. Consideraciones finales: El estudio expuso una crítica sobre la realidad brasileña de los últimos siglos que, completamente invasiva y eurocéntrica, cristaliza la necesidad de proponer una reforma a la educación sanitaria para contemplar el ejercicio de la salud pública en las aldeas indígenas sin dañar los preceptos culturales. ubicaciones.

Palabras clave: Educación para la salud, Salud indígena, Derecho fundamental a la salud.

\section{INTRODUÇÃO}

A educação em saúde é um tema complexo em sua viabilidade, atribuído a vários aspectos: política, filosofia, sociedade, religião e cultura, além dos aspectos práticos e teóricos de indivíduos, grupos, comunidades e sociedades. O conceito de educação em saúde está vinculado aos conceitos de educação e saúde. Tradicionalmente, é entendido como o uso ou não uso das tecnologias mais avançadas para transmitir informações em saúde, e suas críticas têm mostrado suas limitações e não podem explicar a complexidade envolvida no processo educacional.

Conceitos críticos e participativos conquistaram o espaço e compreenderam a educação em saúde para a realização da saúde, considerada uma série de práticas de ensino participativas e emancipatórias que penetram em diversas áreas de atuação e buscam melhorar a sensibilização das pessoas, além de aumentar a conscientização e mobilizar-se para lidar com condições pessoais e coletivas que afetam a qualidade de vida (SALCI MA, et al., 2013).

A prática de educação em saúde envolve três partes dos atores prioritários: profissionais de saúde que valorizam as práticas de prevenção e promoção e tratamento; gestores que apoiam esses profissionais; e pessoas que precisam de construção de conhecimento individual e coletivo e maior autonomia de atendimento.

Embora a definição do Ministério da Saúde proponha elementos que pressupõem essa interação entre as três partes da estratégia para desenvolver esse processo, ainda há uma longa distância entre retórica e prática. Como processo de educação política, a educação em saúde precisa desenvolver pensamento crítico e reflexivo, permitir expor a realidade e propor ações transformadoras, para que o indivíduo tenha autonomia e capacidade de liberação dos sujeitos históricos e sociais, além de propor e fazer recomendações no que concerne cuidar de si, das decisões de saúde da família e da comunidade (FALKENBERG MB, et al., 2014).

A Fundação Nacional de Saúde aprovou o Regulamento no 254 do Ministério da Saúde em 31 de janeiro de 2002, aprovando a Política Nacional de Saúde dos Povos Indígenas, que está relacionada aos subsistemas relacionados à saúde dos povos indígenas no Sistema Único de Saúde (SUS), e esforça-se para garantir que as qualificações culturais dos cidadãos sejam respeitadas, promovendo modelos de saúde diferenciados, exercendo sua cidadania. Dessa forma, é possível superar os fatores de vulnerabilidade dessas populações e combinar suas formas e conceitos tradicionais de saúde com o direito de reafirmar sua cultura (LANDGRAF J, et al., 2019).

Ainda para Landgraf $\mathrm{J}$, et al (2019), os povos indígenas enfrentam dificuldades para alcançar os direitos estabelecidos na Constituição de 1988, que garante seus direitos especiais, e reconheceram suas formas de organização social, costumes, idiomas, crenças e tradições. Em vista do recente reconhecimento dos povos indígenas no Brasil, a saúde indígena ainda possui muitos obstáculos que precisam ser incluídos na agenda 
de discussão e previsão das necessidades de saúde pública do Brasil, e a atenção de várias agências federais é distraída e interrompida para essa tarefa. Além do preconceito causado pela dominação cultural, também devem ser considerados os processos de saúde e doença causados pela desigualdade social ou discriminação étnica (para a população indígena).

Desde 2000, a capacitação e qualificação dos profissionais de saúde brasileiros tem sido objeto de políticas e ações intersetoriais, envolvendo especialmente o Ministério da Saúde e Educação, mas o texto busca refletir sobre a formação e a educação de longo prazo da saúde indígena, enfatizando os profissionais não indígenas. Essa reflexão não é exaustiva, pois busca trazer alguns elementos que contribuem para o debate, destacando os principais pontos e desafios da atenção à saúde indígena, principalmente pela negligência do papel dos povos indígenas nos últimos anos. A educação permanente é parte integrante do espaço de diálogo necessário para entender as diferenças nos processos de saúde/doença/assistência em diferentes partes do país (DIEHL EE e PELLEGRINI MA, 2014).

O objetivo desta revisão é reunir o conhecimento bibliográfico acerca dos aspectos básicos da educação em saúde para profissionais que estarão ligados aos povos indígenas, e a inclusão de políticas educacionais que visem preparar esses profissionais para as diferenças culturais e tradicionais desses povos. Além de reforçar o direito fundamental a saúde, garantia constitucional, para justificar a necessidade de uma educação em saúde mais inclusiva e pautada nos direitos humanos dos grupos étnicos-raciais.

\section{REVISÃO BIBLIOGRÁFICA}

Os profissionais de saúde coletiva sabem que o uso de novas palavras é comum e, inevitavelmente, eles começam a usar novos termos, independentemente de receberem significados diferentes. Novas palavras significam "palavras novas e antigas ou expressões com novos significados". Saúde pública e saúde coletiva, atenção primária e básica, educação em saúde e educação geral em saúde, educação continuada e educação permanente são exemplos dessa ideia.

O Ministério da Saúde define educação em saúde como o processo educacional de construção de conhecimento em saúde voltado para a população. Um conjunto de práticas departamentais que ajudam a aumentar a autonomia das pessoas em enfermagem e debates com profissionais e gerentes, a fim de obter assistência médica de acordo com suas necessidades (FALKENBERG MB, et al., 2014).

Para qualificar o padrão de gestão e a assistência médica, em 2003, foi formulada a Política Nacional de Humanização (PNH) da Atenção e Gestão no Sistema Único de Saúde (HumanizaSUS). Segundo o Ministério da Saúde, a implementação da PNH exige vários eixos de ação e o objetivo desses eixos é institucionalizar, disseminar a estratégia e distribuir principalmente os resultados para a sociedade.

Dentre esses aspectos, destaca-se a educação permanente em saúde e fornece os seguintes princípios norteadores da PNH nesse campo: orientar o processo contínuo de educação em saúde nos próprios serviços de saúde dos trabalhadores; seja inserido Como componente de conteúdo e/ou especializações de cursos de graduação, pós-graduação e extensão relacionados a instituições de treinamento em saúde (MEDEIROS LMOP e BATISTA SHSS, 2016).

Várias barreiras ao acesso dos povos indígenas aos serviços de saúde pública foram descritas como limitadoras aos cuidados de saúde dos povos indígenas tradicionais em diferentes partes do Brasil e do mundo. Entre os principais obstáculos, existem obstáculos organizacionais, geográficos e culturais, incluindo restrições relacionadas à escassez ou falta de intérpretes culturais, que permitam serviços de saúde étnicos e de alta complexidade no Brasil e em diferentes partes do mundo.

No Brasil, o subsistema atencional indígena foi criado como parte do SUS e definido pela Lei oㅜ 9.836, de 23 de setembro de 1999, que trabalhará com a União para utilizar seus próprios recursos. A integração perfeita, financiamento para o subsistema de assistência à saúde do índio e o SUS, promoverão a expressão do subsistema estabelecido por lei com o órgão responsável pela política de saúde aborígine em todo o país (MAIA JA, et al., 2019). 
Os 4 primeiros artigos da lei dispõem que: Art. 19-A. As ações e serviços de saúde voltados para o atendimento das populações indígenas, em todo o território nacional, coletiva ou individualmente, obedecerão ao disposto nesta Lei.

Art. 19-B. É instituído um Subsistema de Atenção à Saúde Indígena, componente do Sistema Único de Saúde - SUS, criado e definido por esta Lei, e pela Lei no 8.142, de 28 de dezembro de 1990, com o qual funcionará em perfeita integração.

Art. 19-C. Caberá à União, com seus recursos próprios, financiar o Subsistema de Atenção à Saúde Indígena.

Art. 19-D. O SUS promoverá a articulação do Subsistema instituído por esta Lei com os órgãos responsáveis pela Política Indígena do País (BRASIL, 1999).

Para Junior DS e Leivas PGC (2017) os direitos sociais básicos dos povos indígenas são exercidos com base em instituições e normas específicas todos os dias, e suas bases e experiência teóricas precisam ser avaliadas e discutidas. Obviamente, as políticas prejudiciais que estão sendo implementadas tiveram um enorme impacto sobre esses direitos, mas pensar em suas próprias ideias é uma tarefa relacionada. $O$ fornecimento do direito à saúde dos povos indígenas é de imensa importância, principalmente porque atualmente possui uma estrutura específica de instituições de saúde que precisam abordar indicadores sociais preocupantes em um contexto multicultural.

Os conceitos de doenças e saúde dos povos indígenas são baseados em fenômenos e relacionamentos diferentes dos conceitos biomédicos ocidentais. Além da sensibilidade dos profissionais da saúde, os conceitos biomédicos ocidentais também precisam ter um diálogo significativo e não impor limites a capacidade de traduzir essas diferenças complexas (JUNIOR DS e LEIVAS PGC, 2017).

\section{Motivos que justificam a necessidade da educação em saúde específica para povos indígenas}

A preservação das comunidades e da cultura indígena tem se tornado um problema recorrente nos tempos atuais. A invasão de madeireiras e exploradores de minérios, além da expansão territorial de agropecuaristas, agroindústrias além de grupos religiosos que buscam disseminar suas crenças nas tribos que resistem no Brasil, colocam em risco a manutenção da cultura e a preservação dos povos que visam manter a forma tradicional de vida. A difusão da educação em saúde na busca de promover uma maior preservação da cultura além de não interferir nos tradicionalismos dessas comunidades, tem como objetivo tornar os profissionais da área mais preparados e menos invasivos (SILVA AB, et al., 2019).

As autoridades públicas brasileiras têm dificuldades em dialogar com os povos indígenas e em reconhecer suas diferenças na prática. O governo planeja ter os mesmos requisitos para os povos indígenas que as organizações não indígenas, porque ele não reconhece suas próprias formas de organização social, formas representativas e maneiras de expressar seus sentimentos e costumes.

A falta de sensibilidade está intimamente ligada ao despreparo dos servidores públicos encarregados de lidar com a causa indígena. A educação em saúde específica para profissionais da saúde que fornecem tratamento nas tribos, busca, além da preservação cultural e manutenção da forma de vida, facilitar o contato e estabelecer diretrizes mais eficazes no combate ao adoecimento desses povos (SILVA DM, et al., 2016).

A diversidade cultural apresenta desafios importantes para os serviços de saúde de todas as sociedades do mundo. No Brasil, para proteger os direitos relacionados à diversidade cultural indígena, foi estabelecido um subsistema de assistência à saúde indígena (SASI) sob a bandeira do tratamento diferenciado, que presta serviços de saúde por meio de uma estrutura separada, mas é parte integrante do SUS, intimamente integrado às práticas tradicionais da comunidade (MOTA SEC e NUNES M, 2018).

Para Almeida MRC (2014), na disseminação do conhecimento e da história dos povos indígenas, as tradições orais os mergulharam quase no folclore. Por volta da década de 1870 , em algumas províncias do nordeste do Brasil, comentários oficiais relataram o desaparecimento dos índios, deixando apenas restos que mantinham certas expressões culturais. 
Manter a cultura ainda existente e preservar as diferenças identificadas é uma obrigação e deve se tornar um objetivo evidentemente programado e passível de investimentos. A saúde dos povos indígenas foi amplamente comprometida com a chegada dos europeus no início do Século XVI, já que doenças as quais a imunidade daqueles povos não estava ambientada, foram espalhadas em todo território invadido, o que tornou ainda mais amplo o genocídio indígena. Ainda hoje, os povos tradicionais brasileiros sofrem com as invasões e a falta de comunicabilidade para preservar suas culturas, além dos adoecimentos que não eram comuns antes da invasão portuguesa, o território está repleto de culturas diversas, e para uma parcela massiva da população, a cultura indígena reduz seu povo a primitivos ou atrasados (PALMQUIST H, 2018).

Somente em 1988, com a promulgação da nova constituição, o Brasil reconheceu os direitos dos povos indígenas como índios, garantindo assim seus direitos coletivos. Desde esse período, muitas mudanças importantes ocorreram no campo da saúde, mas não apenas isso. Deve-se entender que o Brasil ainda é uma das maiores diversidades culturais do mundo, mas, devido às questões já citadas, a maioria dos brasileiros ainda não pode vê-lo. No território do país, existem cerca de 305 povos indígenas que falam 274 idiomas. Estima-se que na região amazônica ainda existam cerca de 70 comunidades que nunca entraram em contato com brancos. As divisões territoriais e culturais de muitos países nessas sociedades não se limitam ao território do Brasil, elas geralmente são distribuídas em mais de um país no continente (BERNI LEV, 2017).

Os povos indígenas no Brasil possuem uma estrutura de saúde complexa e dinâmica, relacionada às características sociais e culturais de cada grupo étnico, e diretamente relacionada ao processo histórico de mudanças sociais, econômicas e ambientais, e o processo histórico está relacionado à população e frente econômica da sociedade em desenvolver níveis nacionais relevantes em diferentes partes do país. Durante séculos, seja através da introdução de novos patógenos que causam graves epidemias, principalmente vírus, esses aspectos tiveram um impacto importante nos determinantes do estado de saúde dos povos indígenas, muito disso se deve a perseguição e morte por exploradores de grupos ou mesmo comunidades inteiras (COIMBRA JR, et al., 2007).

Por isso, devido a sensação clara de injustiça e covardia a qual os povos locais foram e ainda são tratados no Brasil, é que se idealiza as demarcações e se institucionaliza programas com intenções nobres como o de formar profissionais da saúde preparados para lidar com as diferenças étnico culturais nas reservas e aldeias indígenas. A preservação da cultura desses povos é um objetivo que ultrapassa os interesses públicos de buscar meios mais fácil e menos humanistas. Torna-se uma obrigação das novas gerações reparar o descaso e o tratamento que os índios receberam no processo de invasão de suas terras, além de promover uma estrutura que busque conservar sua identidade, fortificar o que for necessário para garantir sua sobrevivência e fornecer de maneira legal subsídios suficientes para a proteção do seu espaço ainda não invadido (SIERING FC, 2008).

\section{A garantia dos direitos indígenas e políticas públicas}

Reconhecer os direitos dos povos indígenas como comunidades, povos ou nações, além dos direitos individuais, também se refere aos direitos coletivos dos povos indígenas. Exortar os países a tomar medidas para ajudar e garantir que os povos indígenas sejam verdadeiramente livres e tenham o livre arbítrio para corrigir ações pretéritas contra seus antepassados, é uma missão jurídica e moral. Também busca-se corrigir erros e injustiças e, quando necessário e desejado pelos povos indígenas, não permitir que os Estados participem das decisões dos povos indígenas em assuntos relacionados a si próprios (GRUBIT S e SORDI A, 2017).

Política indígena refere-se a uma série de ações e atividades realizadas pelo Estado em favor da população indígena que vive em seu território. De acordo com essa lógica, saúde, educação, meio ambiente e desenvolvimento sustentável podem ser considerados como constituindo as políticas departamentais do indígena. A primeira instituição nacional a proteger e assistir os índios criados no início do século passado foi o Serviço de Proteção aos Índios e Localização de Trabalhadores Nacionais (SPILTN), que posteriormente expandiu suas responsabilidades de prestar serviços de saúde aos povos indígenas. E renomeada como Serviço de Proteção ao Índio (SPI), antes da implantação desses órgãos, a Igreja Católica tradicionalmente prestava assistência ocasional aos índios por meio de missionários (MARTINS AL, 2013). 
Os direitos dos povos indígenas estão afixados na Constituição Federal de 1988 sob inúmeros aspectos e várias formas de proteção. Tanto no âmbito da educação quanto nos da cultura, desporto, jurídicos e da saúde. As garantias ocupacionais também são reservadas no texto da Carta Magna, que reservou o espaço ocupado pelos menos como bens da união, e tornou a união a única competente para legislar sobre as terras indígenas. Para que essas terras sejam objeto de pesquisa ou sua finalidade possa ser explorada diferentemente do que apregoa o texto constitucional, compete ao Congresso Nacional autorizar (BRASIL, 1988).

A constituição dispõe em alguns trechos: Artigo 20 - São bens da União: XI - as terras tradicionalmente ocupadas pelos índios.

Artigo 22 - Compete privativamente à união legislar sobre: XIV - populações indígenas;

Artigo 49-É da competência exclusiva do Congresso Nacional: XVI - autorizar, em terras indígenas, a exploração e $o$ aproveitamento de recursos hídricos e a pesquisa e lavra de riquezas minerais;

Artigo 210 - Serão fixados conteúdos mínimos para o ensino fundamental, de maneira a assegurar formação básica comum e respeito aos valores culturais e artísticos, nacionais e regionais. 2. 0 ensino fundamental regular será ministrado em língua portuguesa, assegurada às comunidades indígenas também a utilização de suas línguas maternas e processos próprios de aprendizagem.

Artigo 215 - O Estado garantirá a todos o pleno exercício dos direitos culturais e acesso às fontes da cultura nacional, e apoiará e incentivará a valorização e a difusão das manifestações culturais. 1. O Estado protegerá as manifestações das culturas populares, indígenas e afro-brasileiras, e das de outros grupos participantes do processo civilizatório nacional.

Artigo 231 - São reconhecidos aos índios sua organização social, costumes, línguas, crenças e tradições, e os direitos originários sobre as terras que tradicionalmente ocupam, competindo à União demarcá-las, proteger e fazer respeitar todos os seus bens (BRASIL, 1988).

Segundo o último censo do Instituto Brasileiro de Geografia e Estatística (IBGE, 2010), no Brasil, 890.000 índios vivem em todos os estados, distribuídos em vários estados brasileiros, representando $0,4 \%$ da população brasileira. Esse grupo está distribuído em 505 terras indígenas, representando 12,5\% do território nacional. Portanto, governos de todos os países devem formular normas e políticas de acordo com práticas de participação social para padronizar estratégias para reduzir as desigualdades e desigualdades relacionadas à saúde e refleti-las diretamente no desenvolvimento nacional. Essas iniciativas devem promover um atendimento abrangente com base nas condições locais, incluindo acesso a serviços e atendimento de diferentes profissionais (MENDES AM, et al., 2018).

Para Bernardes AG (2011), o Serviço de Proteção aos Índios (SPI) é a primeira política pública direcionada às populações indígenas, que cria a possibilidade de nacionalização dessas populações e considera a conexão entre trabalho, vida e linguagem como uma abordagem significativa. Essas ações de nacionalização ou formas de governo dos povos indígenas são suas agências: primeiro, a definição de status legal, onde a palavra "Índio" aparecerá como status legal; segundo, se deseja reter militares na aplicação e gerenciamento do SPI.

Nesse sentido, o SPI possui inscrições duplas em práticas importantes no campo das políticas públicas, porque os povos indígenas têm status legal no território nacional. Portanto, o povo indígena passa a ser o foco do investimento político, e a expressão desse status legal no campo militar, ou seja, a nacionalização do povo indígena é feita pelos militares. A atribuição dos índios será um canal e uma maneira intermediária de 
realizar a extinção das entidades distintas dos povos indígenas como e ter diferentes direitos históricos e políticos de autodeterminação será uma maneira de evitar que o racismo nacional continue a funcionar (BERNARDES AG, 2011).

Oliveira LA e Nascimento RG (2012), expõem que essa relação entre políticas indígenas e educacionais se configura de diferentes maneiras em diferentes momentos históricos, mas acredita-se que isso deve ser levado em consideração para considerar o processo de formulação e implementação de políticas educacionais para os povos indígenas. Portanto, ao considerar essa relação, um aspecto que chama atenção é o tratamento da diversidade dos povos indígenas e o tratamento desigual das populações nacionais e regionais em um conjunto de políticas públicas. Entretanto, nem sempre as políticas públicas educacionais voltadas aos povos indígenas agiram de maneira integrativa e na intenção de preservar seus modos de vida.

Portanto, desde as ações dos jesuítas no período colonial até os costumes indígenas do século XX, o principal objetivo da política educacional dos povos indígenas era integrá-los à civilização ou nacionalizá-los. Nessa situação imperativa, assumindo que os índios são inferiores aos brancos civilizados, desta vez no estágio evolutivo primitivo ou selvagem, é necessário estabelecer um campo indígena nas instituições institucionais do país. Fora da era da nação, os índios estavam destinados a desaparecer porque eram sobreviventes de um passado distante (OLIVEIRA LA e NASCIMENTO RG, 2012).

\section{CONSIDERAÇÕES FINAIS}

Conclui-se, portanto, que a promoção e preservação da cultura indígena deve ser tratada com prioridades e muita cautela. Garantir a sobrevivência da identidade desses povos e mesmo assim prestar assistências de saúde, educação e outras garantias públicas é essencial. Devido a isso, a educação em saúde dos profissionais que terão acesso as reservas indígenas, deve ter seus parâmetros cautelosamente elaborados, de forma que não interfira nos direitos e garantias constitucionais reservados a esses povos, à fim de preservar seu modo de vida e também encerrar o ciclo devastador de exploração e dominação iniciado no século XVI pelos invasores portugueses.

\section{REFERÊNCIAS}

1. ALMEIDA MRC. Presença indígena no Nordeste: processos de territorialização, modos de reconhecimento e regimes de memória. Mana, 2014; 20(1): 209-213.

2. BERNARDES AG. Saúde indígena e políticas públicas: alteridade e estado de exceção. Interface - Comunicação, Saúde, Educação, 2011; 15(36): 153-164.

3. BERNI LEV. Psicologia e saude mental indígena: Um panorama para construção de políticas públicas. Psicologia para América Latina, 2017: 64-81.

4. BRASIL, 1988. Constituição da República Federativa do Brasil: promulgada em 5 de outubro de 1988. 4. ed. São Paulo: Saraiva, 1990.

5. BRASIL, 1999. In: PRESIDÊNCIA da República: acrescenta dispositivos à Lei no 8.080, de 19 de setembro de 1990 , que "dispõe sobre as condições para a promoção, proteção e recuperação da saúde, a organização e o funcionamento dos serviços correspondentes e dá outras providências", instituindo o Subsistema de Atenção à Saúde Indígena. Disponível em: http://www.planalto.gov.br/ccivil_03/leis/L9836.htm. Acesso em 16 de jul. 2020.

6. COIMBRA JR, et al. Povos indígenas e o processo saúde-doença. Vol. 1. Rio de Janeiro: Editora FIOCRUZ, 2007; 29p.

7. DIEHL EE, PELLEGRINI MA. Saúde e povos indígenas no Brasil: o desafio da formação e educação permanente de trabalhadores para atuação em contextos interculturais. Cad. Saúde Pública, Rio de Jane, 2014; 30(4): 867-874.

8. FALKENBERG MB, et al. Educação em saúde e educação na saúde: conceitos e implicações para a saúde coletiva. Ciência \& Saúde Coletiva, 2014; 19(3): 847-852.

9. GRUBIT S, SORDI A. Pesquisas nas comunidades indígenas: relações de justiça e igualdade. Boletim - Academia Paulista de Psicologia, 2017; 37(92): 11-23.

10. IBGE. 2010. In: INSTITUTO brasileiro de Geografia e Estatística, População residente, segundo a situação do domicílio e condição de indígena - Brasil 1991/2010. Disponível em: https://indigenas.ibge.gov.br/graficos-e-tabelas2.html. Acesso em: 16 de jul. 2020.

11. JUNIOR DS, LEIVAS PGC. O direito à saúde dos povos indígenas e o paradigma do reconhecimento. Revista Direito e Práxis, 2017; 8(1): 86-117.

12. LANDGRAF J, et al. Desafios para a Educação Permanente em Saúde Indígena: adequando o atendimento do Sistema Único de Saúde no sul do Brasil. Interface - Comunicação, Saúde, Educação, 2019; 24(23): 1-8.

13. MAIA JA, et al. Acesso dos usuários indígenas aos serviços de saúde de média e alta complexidade. DêCiência em Foco, 2019; 3(2): 144-154. 
14. MARTINS AL. Política de saúde indígena no Brasil: reflexões sobre o processo de implementação do Subsistema de Atenção à Saúde Indígena. Dissertação (Mestrado profissional em saúde pública). Escola Nacional de Saúde Pública (ENSP)/Fundação Oswaldo Cruz e ao Instituto de Pesquisa Econômica Aplicada (IPEA), Brasília, $2013 ; 126$ p.

15. MEDEIROS LMOP, BATISTA SHSS. Humanização na formação e no trabalho em saúde: uma análise da literatura. Trabalho, Educação e Saúde, 2016; 14(3): 925-951.

16. MOTA SEC, NUNES M. Por uma atenção diferenciada e menos desigual: o caso do Distrito Sanitário Especial Indígena da Bahia. Saúde Soc., 2018; 27(1): 11-25.

17. OLIVEIRA LA, NASCIMENTO RG. Roteiro para uma história da educação escolar indígena: notas sobre a relação entre política indigenista e educacional. Educ. Soc., 2012; 33(120): 765-781.

18. PALMQUIST H. Questões sobre genocídio e etnocídio indígena: a persistência da destruição. Dissertação (Mestrado em Antropologia). Universidade Federal do Pará, Belém, 2018; 154 p.

19. SALCI MA, et al. Educação em saúde e suas perspectivas teóricas: algumas reflexões. Texto Contexto Enferm, 2013; 22(1): 224-230.

20. SIERING FC. Conquista e dominação dos povos indígenas: Resistência no sertão dos Maracás. (1650- 1701). Dissertação (Mestrado em História Social). Universidade Federal da Bahia, Salvador, 2008; 147 p.

21. SILVA AB, et al. Cultura dos povos originários da floresta amazônica na gestação e no puerpério: uma revisão de escopo sob o ponto de vista da segurança alimentar e nutricional. Saúde Debate, 2019; 43(123): 1219-1239.

22. SILVA DM, et al. Dificuldades enfrentadas pelos indígenas durante a permanência em uma Casa de Saúde Indígena na região Amazônica/Brasil. Saúde Soc., 2016; 25(4): 920-929. 